\title{
Malignant peripheral nerve sheath tumor of temporoparietal region of brain mimicking as glioma: A rare case report
}

\author{
Shivangi Agrawal ${ }^{1}$, Abhinav Kakkar ${ }^{1}$, Ravindra Singh Gothwal ${ }^{2}$, Sandeep Jain ${ }^{2}$, Rohitashwa Dana ${ }^{2}$ \\ From ${ }^{1}$ Junior Resident, ${ }^{2}$ Senior Professor, Department of Radiation Oncology, SMS Hospital, Jaipur, Rajasthan, India
}

\begin{abstract}
Malignant peripheral nerve sheath tumor (MPNST) is exceedingly rare to occur intracerebrally. The incidence of this subtype is $0.001 \%$. Their typical origin is from peripheral nerves of the limbs, trunk, and lumbar and brachial plexuses. It carries a poor prognosis despite aggressive management due to high chances of local recurrence. We present a case of MPNST occurring intracerebrally in a 60-year-old male patient. Imaging showed a $63 \times 74 \times 46 \mathrm{~mm}$ mass lesion in the left temporoparietal region with mass effect. The patient underwent left temporoparietal craniotomy with maximum gross resection of the tumor. Histopathology was suggestive of spindle cell neoplasm. On immunohistochemistry, cells were positive for S-100 and SOX-10, which were consistent with the diagnosis of MPNST. The postoperative contrast-enhanced magnetic resonance imaging of brain showed gross residual disease. Post-operative radiotherapy was given of intensity-modulated radiation therapy followed by chemotherapy of doxorubicin and ifosfamide.
\end{abstract}

Key words: Brain, Malignant, Peripheral nerve sheath, Tumor

$\mathrm{M}$ alignant peripheral nerve sheath tumors (MPNST) are uncommon, accounting for $5 \%$ of softtissue sarcomas [1]. Typically, they originate from nerves of the extremities and trunk or preexisting neurofibromas. Those involving intracranial nerves are highly exceptional. They usually occur de novo or in the setting of neurofibromatosis Type 1 where there is a transition from a plexiform neurofibroma. It is very rare for a schwannoma to transit into MPNST [2]. There is a male predilection with a male to female ratio of 2.5:1 and it mainly occurs between the fourth and sixth decade [3]. The incidence of MPNST is high in patients who have received prior radiation therapy. It is extremely rare for MPNST to occur intracranially. If it occurs, they are highly aggressive with a poor outcome. The incidence of this subtype is as low as $0.001 \%$ and is graded as the WHO Grade IV tumor [4]. The main treatment for MPNST is surgery. Chemotherapy and radiation therapy should be considered for incomplete unresected tumors [5].

We report a rare case of MPNST in a 60-year-old male with MPNST of the left parietotemporal region which is quite an unusual site of presentation. We report this case because of its unique presentation and aggressive multimodality treatment requirement.

\section{Access this article online}

Received - 06 November 2021

Initial Review - 23 November 2021

Accepted - 17 January 2022

DOI: $10.32677 /$ ijcr.v8i1.3295

\section{CASE REPORT}

A 60-year-old male presented to the neurosurgery outpatient department with complaints of progressive headache and vertigo of 1-month duration. The headache was initially mild in severity which had progressed over a period of month to severe. It was dull-aching, continuous, involving predominantly the left temporo parietal region, it was not associated with seizure, visual disturbance, nausea, vomiting and not relieved on taking the over-the-counter analgesics. The vertigo was lasted for around one minute. It was not associated with decreased hearing or tinnitus. There was no history of loss of consciousness.

On examination, the patient was conscious and coherent. On general examination, vitals were stable. Cardiovascular, respiratory, and abdominal examinations were normal. Neurological examination showed no notable abnormalities and there were no signs of Von Recklinghausen disease.

Routine baseline investigations including hematological, renal, and liver function tests were normal. Contrast-enhanced magnetic resonance imaging (CEMRI) brain scan showed a heterogeneous mass lesion approximately $63 \times 74 \times 46 \mathrm{~mm}$ eccentric solid and cystic/necrotic component with moderate perilesional edema of the left parietotemporal hemisphere. It was hypointense on $\mathrm{T} 1$ and heterogeneously hyperintense on $\mathrm{T} 2 \mathrm{~W}$ and the cystic area was mildly hyperintense on T2W/FLAIR. It was causing compression over the left lateral ventricles and midbrain. The pre-operative provisional diagnosis was glioma (Fig. 1).

Correspondence to: Dr. Ravindra Singh Gothwal, Department of Radiation Oncology, SMS Hospital, Jaipur, Rajasthan, India. E-mail: drravindragothwal@ yahoo.com

(C) 2022 Creative Commons Attribution-NonCommercial 4.0 International License (CC BY-NC-ND 4.0). 
Aleft temporoparietal craniotomy was performed with maximum gross resection of the tumor. Post-operative histopathological examination showed many spindle cells arranged in bundles favoring spindle cell neoplasm (Fig. 2). Immunohistochemistry showed that cells were positive for vimentin, S-100, and SOX-10 and negative for glial fibrillary acidic protein (GFAP) and epithelial membrane antigen (EMA) (Fig. 3). The findings were consistent with the diagnosis of MPNST.

Post-operative magnetic resonance imaging (MRI) was done after four weeks which revealed post-operative changes with gliosis and interstitial edema in the left temporal lobe with an area of restricted diffusion which showed patchy enhancement on contrast in favor of residual lesion. Post-operative radiotherapy was given by intensity-modulated radiation therapy of $60 \mathrm{~Gy}$ in 30 fractions. Post radiotherapy MRI brain was done after 1 month which showed gross residual disease. Palliative chemotherapy
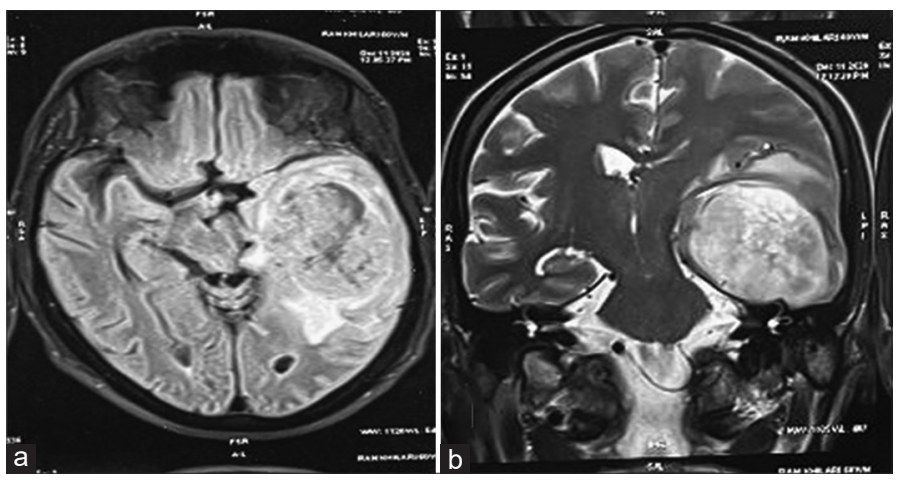

Figure 1: Contrast-enhanced magnetic resonance imaging brain scan shows a heterogeneous mass lesion with moderate perilesional edema in the left parietotemporal hemisphere

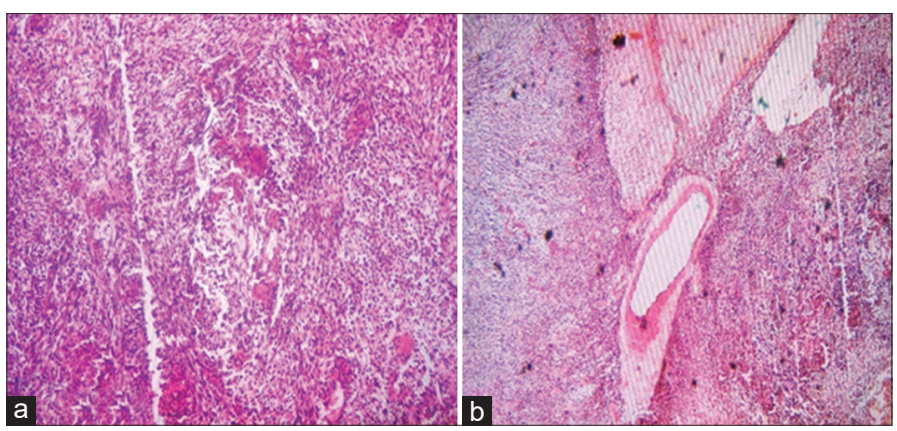

Figure 2: Histopathological examination shows spindle cells in clusters (a) at $40 \times($ b) at $\times 100$

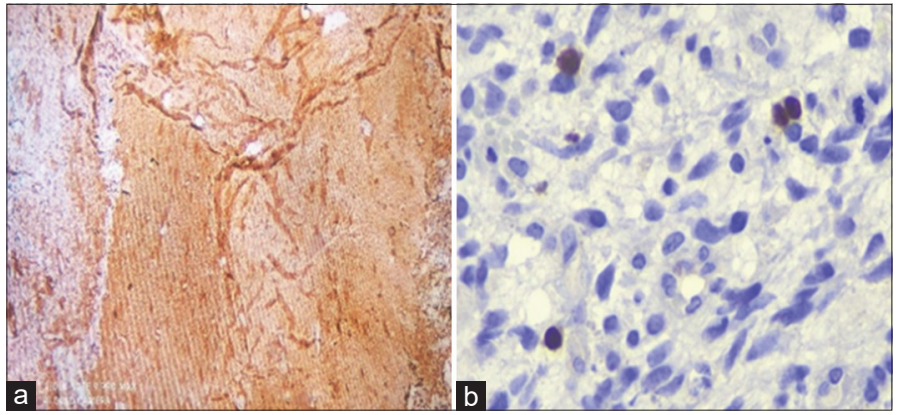

Figure 3: (a) Immunohistochemistry positive for S-100; (b) Cells positive for SOX 10 was planned with ifosfamide and doxorubicin and patient received 3 cycles of chemotherapy. After 3 cycles radiological imaging showed progression of disease, so patient was put on best supportive care.

\section{DISCUSSION}

There are many synonyms for MPNST which include neurofibrosarcoma, malignant neurilemoma, malignant schwannoma, neurogenic sarcoma, and malignant neurofibroma. These synonyms are misleading. To replace previous heterogeneous and often confusing terminology, the World Health Organization gave the term MPNST [6]. More commonly, MPNST is found to arise in the trunk and extremities but rare in the brain, where it is also called malignant intracerebral nerve sheath tumor (MINST) [7].

MINST is believed to arise from aberrant Schwann cells and other mesenchymal cells in the perivascular nerve plexuses around large blood vessels in the subarachnoid space, from adrenergic nerve fibers, innervating cerebral arterioles, in the meningeal branches of the trigeminal nerve, or from the pluripotent cells of neural crest origin [8]. The etiology of MPNST is unknown. Neurofibromatosis gene-NF2 mutations predispose to the development of a schwannoma which can affect the spine and intracranial nerves. While sporadic MPNST is most often seen between the fourth and fifth decades of life, in NF1, they present a decade earlier [9].

Approximately $50-60 \%$ of all cranial MPNSTs are located in the vestibular nerve followed by the vagus, trigeminal, and facial nerves [10]. The clinical manifestations of intracranial MPNST vary with its location and size. Shutran et al. reported a case of trigeminal MPNST with corpus callosum metastasis in which the patient presented with trigeminal neuralgia as the first symptom [11]. If brain parenchyma is involved by MPNST then intracranial hypertension would be the main sign.

MRI is the first investigation of choice for the diagnosis of MPNST. On MRI, the main findings are mixed-signal intensity, irregular contour with necrosis, compression of adjacent brain tissue by the lesions, significant local invasion, and signal enhancement in almost all cases. A computed tomography scan may show tumor invasion of surrounding bone [12]. Two MRI scans of MPNST in the cerebellopontine angle area were reported by Chen et al. with findings of low signal on T1WI and mixed high signal on T2WI along with significant inhomogeneous enhancement [13].

Here, in this case report, we present a 60 -year-old male who had developed headache and vertigo progressive in nature with no neurological deficit. On the CEMRI brain, a heterogeneous lesion was seen in temporoparietal region hypointense on T1 and heterogeneously hyperintense on $\mathrm{T} 2 \mathrm{~W}$ and cystic area was mildly hyperintense on T2W/FLAIR which is consistent with the finding of the literature. Our patient had similar characteristics in terms of cytopathological findings, with previously reported MINST. This included hypercellular spindle cells with marked variation 
in cellular and nuclear size, the presence of syncytial epithelioid tumor cells and multinucleated giant cells, and foci of necrosis. Immunohistochemistry showed positive stains for Vimentin, S100, and Sox 10 and negative for GFAP and EMA ruling out various differential diagnoses and confirming the diagnosis of MPNST.

MPNSTs generally have a poor prognosis with high metastatic potential. The lungs, soft tissues, bones, liver, and brain are all the main documented sites of hematogenous metastases. Metastasis to the spinal cord is more common [3]. Even after complete surgical resection, chances of local recurrence are high ranging in between $30 \%$ and $60 \%$ of cases $[14,15]$.

The standard treatment for MPNST is complete surgical resection. Post-operative radiotherapy is the main adjuvant treatment. The reports suggest that post-operative radiotherapy can improve survival rates and prognosis. The mortality rate with radiotherapy was $65 \%$ while, without radiotherapy, it was $79 \%$, with a 1 -year survival rate of $65 \%$ for the seven survivors who received radiotherapy. It has been reported that combination chemotherapy is effective for the treatment of MPNST. In general, the combination of ifosfamide, etoposide, and anthracycline is used, but the overall remission rate is relatively low. Our patient underwent left temporoparietal craniotomy along with adjuvant radiotherapy. Post radiation treatment MRI scan was done which revealed gross residual disease. The patient was started on ifosfamide and doxorubicin-based palliative chemotherapy. The patient had received three cycles of palliative chemotherapy.

\section{CONCLUSION}

MPNST in the left temporoparietal area is quite rare which requires aggressive multimodality treatment. However, the rate of remission is very low with higher chances of recurrences. The mainstay for radical treatment is surgery and radiation treatment. The cranial MPNST is rarely accessible for gross total resection, and thus carries a poorer prognosis and requires a multi-modality approach. Due to high chances of recurrence, it is of utmost importance to put the patient on close follow-up. In cases of residual disease/recurrences, the multidrug-based regimen has been effective in prolonging progression-free survival, but the remission rate is quite low.

\section{REFERENCES}

1. Minovi A, Basten O, Hunter B, Draf W, Bockmhl U. Malignant peripheral nerve sheath tumors of the head and neck: Management of 10 cases and literature review. Head Neck 2007;29:439-45.

2. Wilkinson JS, Reid H, Armstrong GR. Malignant transformation of a recurrent vestibular schwannoma. J Clin Pathol 2004;57:109-10.

3. Ziadi A, Saliba I. Malignant peripheral nerve sheath tumour of intracranial nerve: A case series review. Auris Nasus Larynx 2010;37:539-45.

4. OzdalB, Murat OZ, Korkmaz E, Ataoglu O, Gungor T, Meydanli MM. Malignant peripheral nerve sheath tumor of the vulva, an unusual differential diagnosis for vulvar mass. Int J Surg Case Rep 2014;5:793-5.

5. Byung SL, Young GK, Dong HK, Mou SL. A long-term survival case of a primary malignant intracerebral nerve sheath tumor. J Korean Neurosurg Soc 2013;54:261-4.

6. Chikkannaiah P, Boovalli MM, Nathiyal V, Venkataramappa S Morphological spectrum of peripheral nerve sheath tumors: An insight into World Health Organization 2013 classification. J Neurosci Rural Pract 2016;7:346-54

7. Chica J, Yepes I, Burks SS, Komotar R, Carrillo R. Case of an intracranial malignant peripheral nerve sheath tumor in the setting of pacer-dependent heart block. Asian J Neurosurg 2018;13:147-9.

8. Wani AA, Laherwal M, Ramzan AU, Malik NK, Lone I, Nizami FA. Malignant peripheral nerve sheath tumor of fourth ventricle and $7-8^{\text {th }}$ cranial nerve complex: Case report. Indian J Cancer 2015;52:578-9.

9. Fox CJ, Tomajian S, Kaye AJ, Russo S, Abadie JV, Kaye AD. Perioperative management of neurofibromatosis type-1. Ochsner J 2012;12:111-21.

10. Scheithauer BW, Erdogan S, Rodriguez FJ. Malignant peripheral nerve sheath tumors of cranial nerves and intracranial contents: A clinicopathologic study of 17 cases. Am J Surg Pathol 2009;33:325-38.

11. Shutran M, Mosbach D, Tataryn Z, Arkun K, Wu JK. Case report: Metastasis of a trigeminal malignant peripheral nerve sheath tumor to the corpus callosum. Neurosurgery 2019;84:E63-7.

12. Nakayama Y, Watanabe M, Suzuki K. Malignant peripheral nerve sheath tumor of the trigeminal nerve: Clinicopathologic features in a young adult patient. Neuropathology 2013;33:541-6.

13. Chen L, Mao Y, Chen H, Zhou LF. Diagnosis and management of intracranial malignant peripheral nerve sheath tumors. Neurosurgery 2008;62:825-32.

14. Nguyen TB, Chin RY, Iqbal F. Malignant peripheral nerve sheath tumor of the spinal accessory nerve. Ann Otolaryngol Rhinol 2016;3:1108.

15. Gupta G, Maniker A. Malignant peripheral nerve sheath tumors. Neurosurg Focus 2007;22:12.

Funding: None; Conflicts of Interest: None Stated.

How to cite this article: Agrawal S, Kakkar A, Gothwal RS, Jain S, Dana R. Malignant peripheral nerve sheath tumor of temporoparietal region of brain mimicking as glioma: A rare case report. Indian $\mathrm{J}$ Case Reports. 2022;8(1):4-6. 